THE ECLIPSING BINARIES IN NGC 5466 AND IMPLICATIONS FOR CLOSE BINARY EVOLUTION 1

\author{
E.F. Milone, and C.R. Stagg \\ RAO, Department of Physics and Astronomy, The University \\ of Calgary, 2500 University Dr., NW, Calgary, Alberta, \\ Canada T2N 1N4 \\ J. Kallrath \\ Astronomische Institute der Universităt Bonn and BASF- \\ AG, D-7600 Ludwigshafen 1, Germany
}

ABSTRACT The three eclipsing binaries among the blue stragglers of the globular cluster NGC 5466 have been modeled. Two of the systems are contact systems and the third is a short-period Algol system. The results indicate that the systems are well on their way to merger, but the implications for the origin of bluestragglers are less clear.

\title{
INTRODUCTION
}

The rarity of binaries in globulars argues for a mechanism that evolves detached binaries into contact systems and ultimately into (spun-down) blue stragglers. The connection between W UMa stars and blue stragglers has been discussed in, e.g., Leonard (1989), while angular momentum loss through magnetic field braking, the likely evolution mechanism, has been amply discussed (e.g. by Webbink 1976, Mochnacki 1981, Rahunen 1981). Mateo et al.'s (1990) discovery of three eclipsing systems in NGC 5466 provides an opportunity to study such systems at late stages of metamorphoses. Their CCD data of NH19, 30, and 31 (for Nemec and Harris 1987) have been modeled with our improved Wilson-Devinney (WD) code (Milone et al. 1992) and an iterating simplex-enhanced version (SWD) (Kallrath et al. 1992) to shorten convergence time and to test model uniqueness.

1 Publications of the RAO, Series B No. 21 . 


\section{MODELING RESULTS}

Modeling details are given by Kallrath et al. (1992); here we briefly summarize the results only.

The significance of NH31 for binary star evolution is underscored by its period: at just over $0.5^{\mathrm{d}}$, it may be the shortest period Algol system detected to date. The first set of trials involved the case of a transit at primary minimum. Both detached and semi-detached solutions are viable. The semidetached option is not viable in the occultation case. The residuals indicate the semi-detached transit case to be slightly preferred over other models; but the issue is moot: RV data especially are needed to decide the issue.

NH19 and $\mathrm{NH} 30$ are contact systems with depth differences and 0'Connell effects in the light curves; Milone et al. (1987, 1991) have demonstrated that spot modeling suffices as a treatment of the asymmetries. The systems are overcontact and very close to their outer Lagrangian surfaces. In NH19, $\iota \approx 74^{\circ}$ indicates partial eclipses, so that the mass ratio cannot be considered secure until confirmed by spectroscopy, also required for $\mathrm{T}_{1}, \mathrm{M}_{1}$, and $\mathrm{M}_{2}$. In $\mathrm{NH}_{3} \mathrm{O}$, a large uncertainty in $\mathrm{q}$ indicates that this parameter was not well determined and more data are needed here also. The optimized $D C$ and simplex solutions differed slightly for this system, probably partially due to the condition that the minima of parameter space are relatively shallow. Fig. 1 shows system representations.
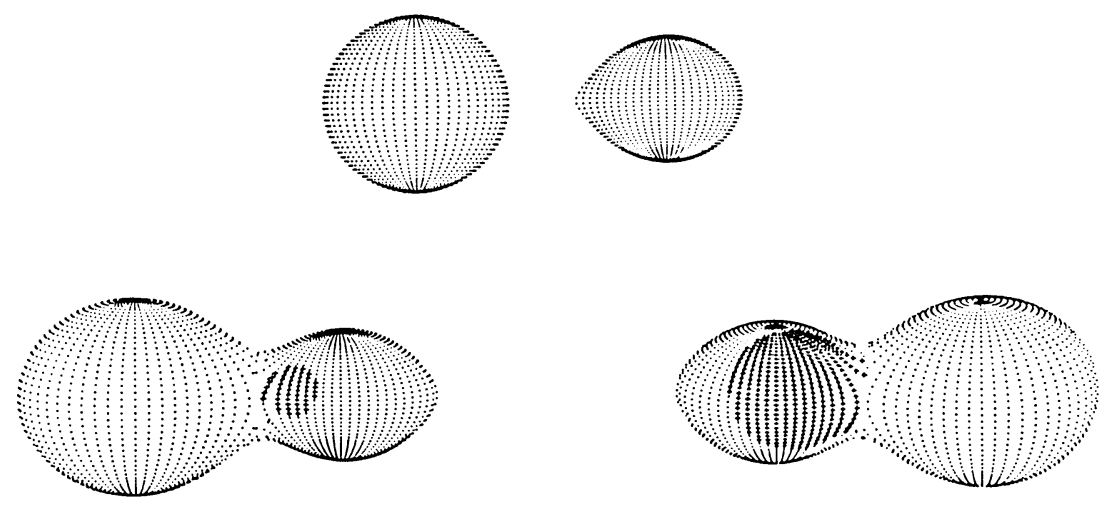

Fig. 1 Adopted models of NH31, (top), NH3O (left), (both at $\phi=0.25$ ) and $N H 19$ (right) at $0.75^{\mathrm{p}}$, via Binary Maker, a software package produced and distributed by D.H. Bradstreet. 


\section{DISCUSSION}

Our solutions differ slightly from the analyses given by Mateo et al. (1990): $\mathrm{T}_{2}$ in the $\mathrm{W}$ UMa systems $\mathrm{NH} 19$ and $\mathrm{NH} 30$ are different from $\mathrm{T}_{1}$ as suggested by the depth differences in the minima. Mateo et al.'s estimates of $q(0.10 \pm 0.04$ and $0.14 \pm$ 0.04 for $N H 19$ and $N H 30$, respectively) were determined from statistical properties of population I field W UMa stars with relatively undistorted light curves and they assumed the radius of the inner contact surface as the radius of each component. Although the work reported here is not dependent on these assumptions, the models indicate that the components of NH19 and $\mathrm{NH} 30$ are very close to their outer Lagrangian surfaces, with contact parameters $\sim 0.94$ so that that mass loss can have occurred. The computed distances for the individual systems show small discrepancies $(\leq 2 \sigma)$, when compared to the distance derived by Nemec and Harris (1987). In particular, NH3O may be a background system, since $\left(\mathrm{V}-\mathrm{MV}_{\mathrm{V}}\right)_{\mathrm{c}}=21.5 \pm 1.9$, compared to Nemec and Harris' (1987) value, 16.0; alternatively, the components may be undermassive.

The binaries are among the blue stragglers of the cluster. Mateo et al. argue that the merger mechanism should function only in relatively old clusters, where the turn-off mass is less than about 2 or $3 \mathrm{M}_{\odot}$, because, according to Iben and Tutukov (1984), this is the upper mass limit for strong magnetic fields which are needed to provide the magnetic braking mechanism for angular momentum loss. The presence of the $0^{\prime}$ Connell effect in two of the three systems studied here is supportive of that view, because surface brightness variation across the disk of a component due to stellar active regions is a possible mechanism for the 0'Connell effect, which is seen in other A-type systems also.

Mateo et al. predict that between 38 and 158 of all population II blue stragglers should be detectable contact binaries. While the evolutionary merger process can yield blue straggler-like products, it is also true that mergers can be greatly aided if not accomplished through binary-binary collisions, especially in cluster cores. It is also probable that blue stragglers can be formed in still other ways (see Leonard 1989). It is interesting that in our preferred models, the hotter components themselves fall into the blue-straggler region of the CMD (see Fig. 2); therefore, the duplicity in itself cannot explain the blue-straggler condition.

B.J.A. Sugars assisted with the modeling. This work was supported by NSERC and University of Calgary research grants to EFM, a Grant from GKSS of Germany for Kallrath's travel, and a University of Calgary Postdoctoral Fellowship to CRS. 


\section{REFERENCES}

Eggen, 0., and Iben. I. 1989, AJ. 97, 431.

Iben, I., and Tutukov, 1984. ApJ. 284, 719.

Kallrath, J., Milone, E.F., and Stagg, C.R. 1992, ApJ., in press.

Leonard, P.J.T. 1989, AJ. 98, 217.

Mateo, M., Harris, H.C., Nemec, J., and Olszewski, E.W. 1990, AJ. $100,469$.

Milone, E.F., Groisman, G., Fry, D.J.I.F., and Bradstreet, D.H. 1991, ApJ. 370, 677.

Milone, E.F., Hrivnak, B,J, and Wilson, R.E. 1987, ApJ. 319, 325.

Milone, E.F., Stagg, C.R., and Kurucz, R. 1992, ApJ. Supp1., in press.

Mochnacki, S.W. 1981, ApJ. 245, 650.

Nemec, J., and Harris, H.C. 1987, ApJ. 316, 172.

Rahunen, T. 1982, A\&A. 102, 81.

Webbink, R. 1976, ApJ. 209, 829.

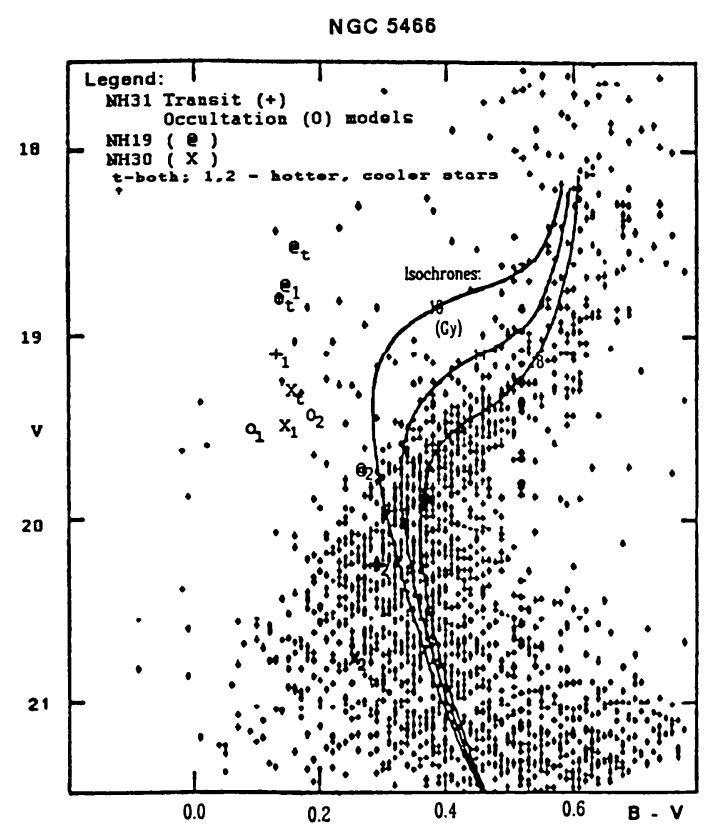

Fig. 2 The CMD of NGC 5466 (adapted from Nemec and Harris 1987). The more luminous components are themselves seen to lie in the blue-straggler region (upper left) of the cluster; also shown are their isochrones for ages of 10, 14, and $18 \mathrm{~Gy}$. 